\title{
Prediction of flood using optimized neural network with Gray Wolf Algorithm (Maroon River case study)
}

\section{Sajjad Amiri Doumari ${ }^{1}$ (i)}

${ }^{1}$ Department of Mathematics and Computer science, Sirjan University of Technology, Sirjan, Iran

*Correspondence: amiri.sajjad@hotmail.com; amiri@sirjantech.ac.ir; Tel: +98-913-340-3952 (F.L.)

Keywords: Flood Routing, Artificial Neural Network, Gray Wolf Algorithm, Maroon River

\begin{abstract}
Floods, as one of the most frequent natural hazards, cause irreparable damage to infrastructure and agriculture, and housing every year. In order to avoid financial and human losses, the importance of flood forecasting seems inevitable. Considering that floods are caused by many natural and anthropogenic factors and also have limitations such as lack of complete information. In this study, artificial neural networks have been used as an efficient method for flood prediction. The neural network inputs include the Dubai River and the Eshel River, this data was collected over 8 Years from the Maroon River. The network used is a multilayer perceptron, also the neural network weights are optimized by the Gray wolf algorithm and the results are compared with other common methods. Analysis of the output results shows that the neural network with the Gray Wolf algorithm has better results than neural network and Genetic algorithms and the error of this method is $0.53 \%$, which indicates high accuracy and precision for flood prediction compared to other evolutionary algorithms. This method is used to obtain the best amount of data for testing and training. As the results, the best rate is $80 \%$ for training and $20 \%$ for testing. Obtained results show the neural network error squares with $80 \%$ of the training data and $20 \%$ of the test data.
\end{abstract}

\section{Introduction}

Flood is the second natural disaster that causes great damage to human societies every year. Among these, cities and population centers have the highest risk and probability of tangible physical damage due to floods (Firoozeh, 2014; Abiodun et al., 2018). Other losses can hardly be considered monetary value. And we can point to the environmental changes that take place by floods, such as water pollution, erosion, and its social aspects (Adib, 2021). Moreover, Urbanization leads to land coming out its natural state. Maybe it becomes an economically and demographically developed area using agricultural industry. In this process, major changes occur in natural environments. Urban development will have many negative effects on the quality of water resources as well as on aquatic and arid ecosystems. This work is done by other groups (Ehteram, 2021; Melesse, 2021). It is also one of the influential factors of the flood (Firoozeh, 2014). 58\% of all-natural disasters and 33\% of economic losses were caused by floods. Most of these casualties have occurred in developing countries (Berz, G., 2000). The first flood prediction in the modern world was made in 1854 in France. This year, Bell Grand predicted the flood three days ago using the telegraph and the 
very simple relationship between rising levels in the tributaries and rising levels of the Seine River in Paris (Berz, 2000). This prediction led to a reduction in casualties and financial losses (Aoulmi, 2021). Since then, we have seen tremendous progress in the field of communication systems and flood forecasting and warning systems. There is an internationally useful experience in building advanced flood forecasting systems, both in terms of hardware and software. The components of existing flood warning systems include three main parts, which are (Heydari et al., 2005):

- Measure, monitor, and send data

- Software section and mathematical models

- Management and information department

The most important components of the system software include rainfall-runoff simulation and formulation of governing relationships in the rainfall-runoff conversion process. Hydrological forecasts and warnings are used for many purposes. From predicting short-term events such as flash floods to seasonal forecasts for water supply, irrigation, energy production or shipping in rivers, forecasting techniques, and from simple experimental relationships and correlation relationships to the use of complex mathematical models involving all stages of river flooding, is variable (Ramezani, 2021). The value of a prediction largely depends on the accuracy of the prediction (Heydari et al., 2005). In recent years, researchers have done extensive research on flood prediction and the factors and ways to prevent it. The following is a review of articles on this topic (Yadav, 2021). Bui et al. (2016) have worked on a combined artificial intelligence method based on neural network and fuzzy inference model and meta-heuristic optimization for a flood. The sensitivity of high-frequency tropical storm modeling using GIS has been simplified using neural networks, fuzzy systems, and meta-heuristic optimization for flood prediction. And used the Genetic Algorithm (GA) and the Particle Swarm Algorithm (PSO) to optimize. The mean squared error, the mean absolute error, the Receiver operating characteristic (ROC), and the area below the receiver operating characteristic curve were used to evaluate performance and predictability (Zhang, 2021). They also compared support vector machines (SVM) with decision tree methods, that the optimized non-fuzzy method has provided a better answer (Bui et al., 2016).

Adib and Mahmoodi (2016) have worked on the prediction of suspended sediments in floods using neural networks and genetic algorithms and Markov chains. In fact, measuring the amount of suspended sediment is a difficult task for engineers in flood conditions. Using the data of the last 42 years, they have trained the perceptron neural network. They used the Lundberg-Markov method to train the neural network, and to reduce the error, they used the mean squared error and increased the correlation coefficient R. Next, the neural network parameters are optimized with a genetic algorithm. That optimization with genetics is obtained according to the result. Where it can reduce the average error squares by up to $42 \%$ (Adib et al., 2017). Haddad et al. (2016) used a self-tuning neural network method to predict surface currents. In this paper, the variables of temperature effectiveness and precipitation evapotranspiration as well as genetic algorithms are used to optimize the variables for prediction. Its purpose was to minimize the mean square error. The results showed better performance with the genetic algorithm. The number of layers also affected the results of the neural network (Bozorg-Haddad et al., 2016). Ramezani et al. (2015) worked on the prediction of river sediment using the artificial feed network model. They obtained the information based on the flow and height of a river in southern Iran, and implemented the neural network, and optimized the network weights using a population-based algorithm, which achieved good results in the mean square error (Ramezani, 2011).

Table 1 examines in more detail the parameters that have been studied in most articles for flood prediction. So that they can be easily examined, and which parameters 
and evaluation methods can be used to achieve a more effective result. This study aims to predict the occurrence of floods and prevent flood damage, which has been done by using artificial neural networks as an efficient method. Bui et al and his co-workers proposed an algorithm that combined methods of neural networks such as fuzzy, genetics, particle swarm, and decision tree. They used the algorithm on 76 million floodprone areas of Vietnam, in other words, 30 million images. The parameters used in their study are slope, height, curvature, moisture horn, distance to the river, and current density. They evaluated average squares error, mean absolute error, performance specifications of the recipient, and the surface below the receiver profile curve for their study. Eventually, its predictability is $91.1 \%$ was achieved and its average error squares are 0.0306 (Bui et al., 2016).

Soomlek et al., 2015, and his team studied the use of the backpropagation neural network to predict floods in Thailand. They used a neural network algorithm on 278 samples from 2008 to 2010 . The used parameters in their study are flow rate, rainfall effects, the water level in nearby dams, and the amount of free water. They evaluated average squares error, correlation coefficient, and productivity. The predictability in their method is $90 \%$ and its average error squared is 0.1139 (Soomlek et al., 2015).

Melesse et al., 2011, and his team studied a neural network model for flood simulation using GIS: Johar River Basin in Malaysia. They used a multilayer perceptron neural network algorithm on 267 samples. The used parameters in their study are rainfall, height, focus time, and soil science. They evaluated average square error, average squares error, the sum of the error squares, coefficient per determination, average square error, also average squared. The achieved mean square error in their work is 0.93 . Also, they studied the prediction of suspended sediment load in the river using an artificial neural network. They used a multilayer perceptron neural network algorithm on 247 data. Discharge, discharge the day before, rainfall, and sediment are the used parameters in their study. The nonlinear regression model and time series model were used to evaluate the accuracy of their work (Melesse et al., 2011).

Adib et al. and Mahmoodi et al., 2017, studied the prediction of suspended sediments in floods using a neural network and genetic algorithm and Markov chain on the obtained data from 1968 to 2009 (Adib et al., 2017). The used parameters in their study were sediment concentration, discharge, the relationship between sediment and sedimentation. The mean normal square error and correlation coefficient are evaluated in their work. The average normal square error in their study is improved by up to $80 \%$ (Adib et al., 2017).

Bozorg-Haddad, 2016, and his team studied the prediction of surface flows by the neural network method (Bozorg-Haddad et al., 2016). In addition to the neural network method, Genetics was used in their work. The 1200 samples, the collected data is for 10 years, have been examined. The used parameters in their study are temperatures, evaporation, sweating, and rainfall. Average squares error is evaluated in their work. The reported average error squares were 0.0125 (Bozorg-Haddad et al., 2016).

Flood prediction using a feedforward neural network was studied by Bakhtiari et al and his team. They used a neural network algorithm on 615 cases which were collected from 2010 to 2011. River flow and the amount of rainfall are the used parameters in their study. They evaluated average square deviation, productivity coefficient, error from peak discharge, and error from peak time. The obtained accuracy in their study is $91 \%$ (Bakhtiari et al., 2010). In recent years, with the emergence and development of computational intelligence, which is actually the extraction of intelligence, knowledge, and algorithm of numerical computation and the provision of up-to-date numerical data, a lot of interest has been created among researchers. As it has been introduced in different sciences and has many applications. The main components of computational 
intelligence include neural networks and fuzzy logic, that artificial neural networks simulate the structure and function of natural neurons and fuzzy logic of approximate inference (Heidari et al., 2016). According to most researchers, the neural network performs well in analyzing unknown and complex issues for two main reasons. First, it has a good relationship between data input and output with pattern recognition. Also, compared to other models, it shows less sensitivity to the existence of errors in the input information. The reason for this is the distributive processing of information in it. And instead of imposing the entire computation load on one processor unit, a large number of processor units are implemented simultaneously. As a result, by reducing the share of each of them, it does not have much negative impact on network performance and output (Latt, 2015).

\section{Methods}

The perceptron network is one of the most famous neural networks and their multilayer state is one of the most widely used neural networks. This neural network is based on a computational unit called the perceptron (see Fig. 1). Perceptron networks probably had the greatest impact on early neural networks. Perceptron learning law is stronger than Hubb law. And with the right assumptions, it can be shown that this learning, along with repetition, converges to the correct weights. Convergence means that network learning leads to weight estimates, which allows the network to generate the correct output value for each of the training input patterns (and similar patterns). A perceptron takes vectors of inputs with real values and calculates a linear combination of inputs. If the threshold value is higher, the perceptron output will be equal to 1 , otherwise, it will be equal to -1 . Perceptron neural networks, especially multilayer perceptron, are among the most functional neural networks. These networks are able to perform a nonlinear mapping with the desired accuracy by properly selecting the number of layers and nerve cells, which are often not many (Abiodun et al., 2018).

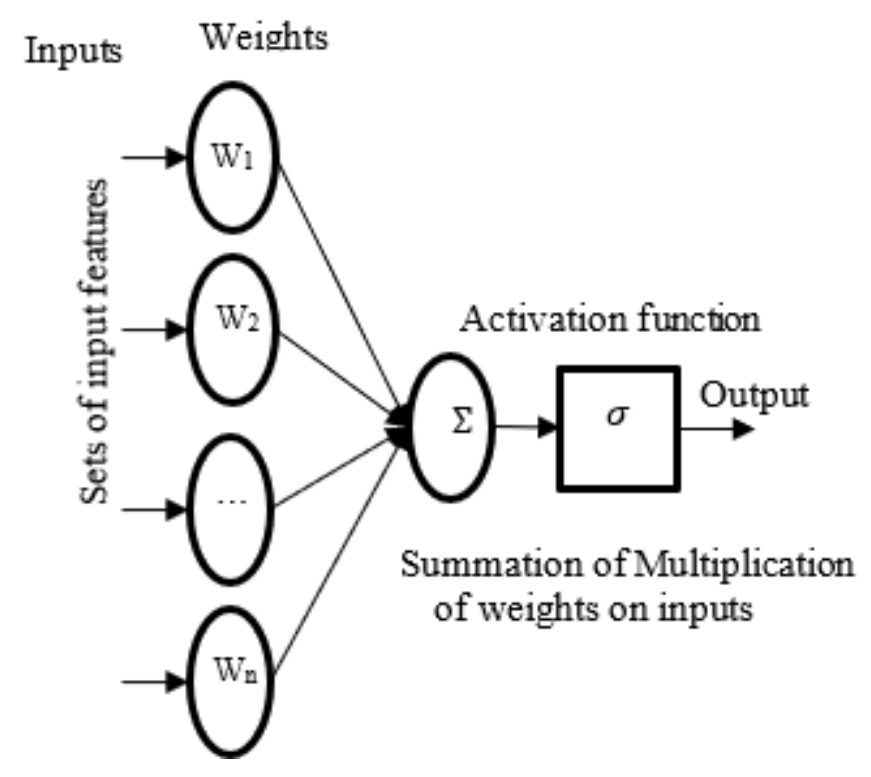

Figure 1. View of the multilayer perceptron network (Abiodun et al., 2018)

In addition to the structure of the network, the method of determining the values of the weights that it is taught is an important distinguishing feature among different neural networks. Training in neural networks, like many other techniques, can be of both 
supervised learning and unsupervised learning types. In addition, there are networks that are fixed in weight and do not require training and repetition.

The standard Gray wolf optimization algorithm is one of the newest evolutionary algorithms. It is inspired by the leadership hierarchy structure and social behavior of wolves while hunting and has been proposed by Mirjalili et al. (2014). Most Gray wolves prefer to live in a group with an average of 5 to 12 wolves. They have strict rules in the social hierarchy. According to their study, the group of Gray wolves includes four species:

1. Alpha $(\alpha)$ wolves: lead the group. Alpha wolves are responsible for making decisions that are made in the group.

2. Beta wolves $(\beta)$ include wolves that help alpha wolves make decisions and other activities. Beta wolves can be male or female, these wolves are the best candidate for being alpha.

3. Omega $(\omega)$ wolves: play the role of the victim. Omega wolves must always surrender to other wolves, and they are the last wolves allowed to eat.

4. Delta Wolves $(\delta)$ : they must surrender to beta and alpha wolves. But they dominate omega wolves. Scouts, officers, elders, hunters, and guards belong to this category. The Scouts are responsible for observing the boundaries of the territory and warning the group in times of danger. Officers guarantee the security of the group and protect the group. Elders are experienced wolves that have previously been beta and alpha. Predators are beta and alpha wolves that help the group hunt prey and provide food. Finally, the guards are responsible for the injured, sick, and weak wolves in the group (Mirjalili et al.,2014).

In the grey wolf optimization (GWO) mathematical model, the optimal solution is called a. The second and third optimal solutions were $\beta$ and $\delta$, respectively. Other candidate solutions are also assumed to be $\omega$. The GWO algorithm uses three solutions $a, \beta$, and $\delta$ to guide the hunt and the $\omega$ solutions follow these three. In order to hunt, $a$ group of wolves encircles the prey. The following equations (1), (2), (3), and (4) are used to simulate the encircling prey behavior (Mirjalili et al. (2014).

$$
\vec{X}(t+1)=\vec{X}_{p}(t)+\vec{A}, \vec{D}
$$

where $\mathrm{t}$ is the number of iterations, $\vec{A}$ and $\vec{C}$ are coefficient vectors, $\vec{X}_{p}$ is the position of the prey, and $\vec{X}$ is the position of the Gray wolves, as $\vec{D}$ is defined in Equation (2) (Mirjalili et al., 2014).

$$
\vec{D}=\left|\vec{C}, \vec{X}_{p}(t)-\vec{X}(t)\right|
$$

The vectors $\vec{A}$ and $\vec{C}$ are calculated by Equations (3) and (4) (Mirjalili et al., 2014).

$$
\begin{gathered}
\vec{A}=2 a, \vec{r}_{1}-a \\
\vec{C}=2 \vec{r}_{2}
\end{gathered}
$$

where component decreases linearly from 2 to 0 during the repetition period. $r_{1}$ and $r_{2}$ are random vectors in the range $[0,1]$. Hunting is usually led by alpha wolves. Beta and delta wolves may also occasionally hunt. In order to mathematically model the Gray wolf hunting behavior, we use a (best candidate solution), $\beta$ (second-best candidate solution), and $\delta$ (third-best candidate solution) assuming they have the best knowledge of prey position. Thus, we keep the three best answers we have so far. And we force other search engines such as Omega to update their position according to the position of the best search agents. We use Equation (5) to update the position of the wolves. 


$$
\vec{X}(t+1)=\frac{\vec{X}_{1}+\vec{X}_{2}+\vec{X}_{3}}{3}
$$

Where $\vec{X}_{1}, \vec{X}_{2}, \vec{X}_{3}$ are defined in Equations (6), (7), and (8).

$$
\begin{aligned}
\vec{X}_{1} & =\left|\vec{X}_{\alpha}-\vec{A}_{1}, \vec{D}_{\alpha}\right| \\
\vec{X}_{2} & =\left|\vec{X}_{\beta}-\vec{A}_{2}, \vec{D}_{\beta}\right| \\
\vec{X}_{3} & =\left|\vec{X}_{\delta}-\vec{A}_{3}, \vec{D}_{\delta}\right|
\end{aligned}
$$

where $\vec{X}_{\alpha}, \vec{X}_{\beta}, \vec{X}_{\delta}$ the position of the first three best solutions in congestion is repeated in at. $\vec{A}_{1}, \vec{A}_{2}, A_{3}$ of Equation (3) and $\vec{D}_{\alpha}, D_{\beta}, \vec{D}_{\delta}$ are defined in Equations (9), (10) and (11), respectively.

$$
\begin{aligned}
& \vec{D}_{\alpha}=\left|\vec{C}_{1}, \vec{X}_{\alpha}-\vec{X}\right| \\
& \vec{D}_{\beta}=\left|\vec{C}_{2}, \vec{X}_{\beta}-\vec{X}\right| \\
& \vec{D}_{\delta}=\left|\vec{C}_{3}, \vec{X}_{\delta}-\vec{X}\right|
\end{aligned}
$$

where $C_{1}, C_{2}, C_{3}$ are defined in Equation (4). The final note on GWO is the parameter used in Equation (3). Which is updated to control the trade-off between exploration and exploitation. Parameter $a$ is updated linearly from 2 to 0 each time according to Equation (12).

$$
a=2-t \frac{2}{\text { MacIter }}
$$

Where $t$ is the number of iterations and Maciter is the total number of iterations allowed for optimization. Finally, Standard Gray Wolf Optimization (GWO) Algorithm 1 is described as follows:

Algorithm 1- Standard Gray Wolf Optimization Algorithm (GWO)

input: $n$ Number of gray wolves in the pack, $\mathrm{N}_{\text {Iter }}$ Number of iterations for optimization.

output: $x_{\alpha}$ Optimal Gray wolf position, $\mathrm{f}\left(x_{\alpha}\right)$ Best fitness value

1. Initialize a population of $\mathrm{n}$ Gray wolves positions randomly.

2. Find the $\alpha, \beta$ and $\delta$ solutions based on their fitness values.

3. while Stopping criteria not met do

foreach wolf $_{i} \in$ pack do

Update current wolf's position according to Eq (5).

\section{end}

I Update a , A and $C$

II Evaluate the positions of individual wolves.

III Update $\alpha, \beta$ and $\delta$

end

4. Return $\alpha$ 
In general, information from two stations is required to predict floods. One station is in the upper part of the river and the other station is in the lower part of the river. Also, two parameters of discharge and river scale are used for the neural network. For this purpose, the number of Gray wolves and population of Gray wolves are considered. The data used in this study to predict floods include 2,500 items (number of iteration) obtained from the Maroon River. This data is between the years 1389 to 1396 , which is given in Table 1 , the names of the stations, and the number of data of each station in each year.

Table 1. Measuring station information

\begin{tabular}{ccccc}
\hline Station & Year & Number of patterns & Year & Number of patterns \\
\hline \multirow{3}{*}{ Kheirabad } & 2009 & 269 & 2014 & 339 \\
Flora bridge & 2011 & 274 & 2015 & 330 \\
& 2012 & 364 & 2016 & 335 \\
& 2013 & 345 & 2017 & 346 \\
\hline
\end{tabular}

However, some data has been deleted due to a problem with the parameter type. Data consists of 6 input parameters and 1 output parameter. Input parameters include river scale on the same day, one day before, and two days before at Kheirabad station. Also, the river discharge has been used on the same day, one day before and two days before at Kheirabad station. Also, the output parameter includes the flow of the Flora bridge.

The scale is used to measure water levels in various water projects, including rivers (with 40 meter width), lakes (with 18 meter width), dams (with 15 meter width), canals (with 10 meter width), pools (with 6 meter width), and more. River discharge is actually the volume of water that passes through a specific section of the river in a unit of time. The average discharge of a river in a given period is presented. Also, the minimum discharge is the amount of river water in the driest times of the year and the maximum discharge is the maximum river discharge in the wettest times of the year. The discharge unit in this study is cubic meters per second.

The Moore neural network used in this study is a 2-layer perceptron neural network. First, the data is normalized between bases 1 and -1 , which is the normalization of the data (Seyed Abbasi et al.,2011). Neural network models have been widely used in a variety of applications. Error back-propagation networks are the most widely used in artificial neural networks to solve a large number of real problems. Neural network design involves selecting an optimal set of design parameters. To achieve fast convergence during training and the accuracy required when calling. The accuracy of any training approximation depends on the selection of appropriate weights for the neural network. Unfortunately, the error back-propagation network is a local search algorithm. Therefore, it causes it to be located in the local trap. If the initial weights are on a local slope, the algorithm will probably be in a local optimum. Researchers use different methods to set these parameters correctly for each problem. For this purpose, the Gray wolf algorithm has been studied and used in this realm. Using the Gray wolf algorithm, which has a high memory and time complexity to find the appropriate initial values of weights as well as other parameters. Which varies depending on the dimensions of the issue. In this paper, we use the Gray wolf algorithm to try to determine the best parameters for the neural network in each problem. The performance function in this method is the mean squared error in neural network training. And the goal is to find the appropriate set of basic neural network parameters to achieve the minimum mean error. 


\section{Results}

Considering the previous section and utilizing artificial neural network, here the obtained results are provided. In this study, artificial neural networks have been used as an efficient method for flood prediction. The neural network inputs include the Dubai River and the Eshel River, this data was collected over 8 Years from the Maroon River. The network used is a multilayer perceptron, also the neural network weights are optimized by the Gray wolf algorithm and the results are compared with other common methods. Analysis of the output results shows that the neural network with the Gray Wolf algorithm has better results than neural network and Genetic algorithms and the error of this method is $0.53 \%$, which indicates high accuracy and precision for flood prediction compared to other evolutionary algorithms. Sensitivity, Specificity, and system performance characteristic curves were used for evaluation. In fact, these two parameters are used to evaluate a binary classification experiment. When data can be divided into positive and negative groups or zero and one. The accuracy of the results of an experiment that divides information into two categories can be measured and described using sensitivity and specificity indicators. Sensitivity is the likelihood that a person with a particular trait will be identified by the same tool. In other words, sensitivity is the ability of the test to detect positive cases. And is the probability that a person without a particular characteristic will be identified by that measuring instrument without that characteristic. Sensitivity and specificity are statistical measures that are used to measure the accuracy and validity of a test incorrectly identifying the desired Specificity (Akobeng,2007). Table 2. Shows the sensitivity and specificity.

Table 2. General table of sensitivity and Specificity

\begin{tabular}{|c|c|c|c|}
\hline & & Prediction & ass \\
\hline \multirow{3}{*}{$\begin{array}{l}\text { Real } \\
\text { class }\end{array}$} & & $\begin{array}{c}\text { Positive } \\
\text { class }\end{array}$ & $\begin{array}{c}\text { Negative } \\
\text { class }\end{array}$ \\
\hline & \multirow{2}{*}{$\begin{array}{c}\text { Positive } \\
\text { class } \\
\text { Negative } \\
\text { class }\end{array}$} & $\begin{array}{c}\text { True } \\
\text { Positive }\end{array}$ & $\begin{array}{c}\text { False } \\
\text { Negative }\end{array}$ \\
\hline & & $\begin{array}{c}\text { False } \\
\text { Positive }\end{array}$ & $\begin{array}{c}\text { True } \\
\text { Negative }\end{array}$ \\
\hline
\end{tabular}

$$
\begin{aligned}
\text { Sensitivity } & =\frac{\text { True Positive }}{\text { True Positive }+ \text { False Negative }} \\
\text { Spectificity } & =\frac{\text { True Negative }}{\text { True Negative }+ \text { False positive }} \\
\text { Accuracy } & =\frac{\text { True Positive }+ \text { True Negative }}{\text { True Negative }+ \text { False Positive }+ \text { True Positive }+ \text { False Negative }}
\end{aligned}
$$

The previous section described the neural network and detailed Gray wolf algorithms. And it was said that in order to optimize the neural network, the Gray wolf algorithm was used. In the following, we will express the results of the combination of neural network and Gray wolf.

In this research, the evaluation method is categorized as follows. Which has two classes, that floods happen or floods do not happen. True Positive: Recognize that a flood is coming and that it does really come a flood. False Positive: Recognize that a flood is coming and that it does not really come a flood. True Negative: Recognize that 
a flood is not coming and that it does not really come a flood. False Negative: Recognize that a flood is not coming and that it does really come a flood.

\section{Discussion}

As mentioned, the main structure used in this study is a 2-layer perceptron neural network. The following are the results of the evaluation of the network performance in the form of tables. This method is used to obtain the best amount of data for testing and training. As the results, the best rate is $80 \%$ for training and $20 \%$ for testing. Obtained results show the neural network error squares with $80 \%$ of the training data and $20 \%$ of the test data.

Table 3 examines the results of the test and training data. This method is used to obtain the best amount of data for testing and training.

Table 3. The validity of assessment with different amount of test and training data for neural network

\begin{tabular}{cccc}
\hline MSE test & MSE train & Train data & Test data \\
\hline 0.0066 & $1.704^{-4}$ & $80 \%$ & $20 \%$ \\
0.0067 & $1.8731^{-24}$ & $70 \%$ & $30 \%$ \\
0.0078 & $1.8427^{-4}$ & $60 \%$ & $40 \%$ \\
0.0138 & $5.2331^{-5}$ & $50 \%$ & $50 \%$ \\
\hline
\end{tabular}

As the results show, the best rate is $80 \%$ for training and $20 \%$ for testing. Figure 2 shows the neural network error squares with $80 \%$ of the training data and $20 \%$ of the test data.

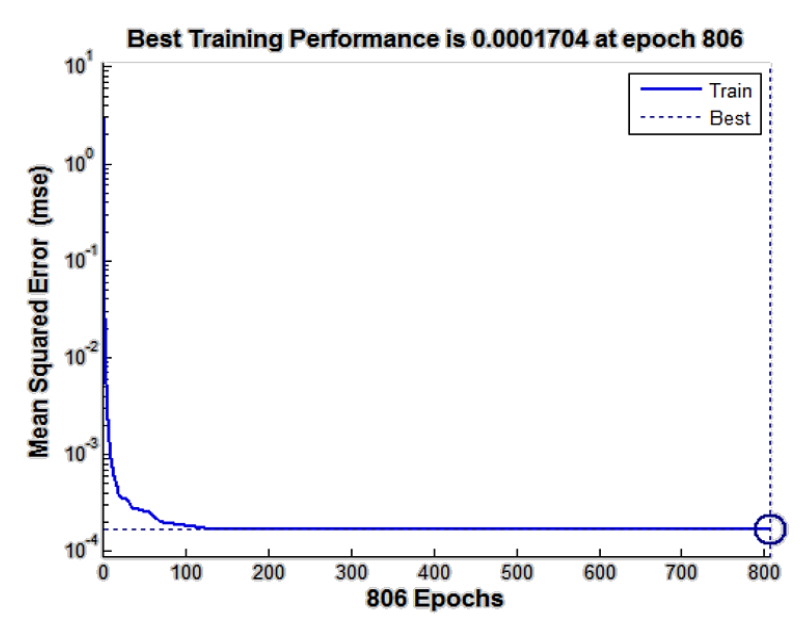

Figure 2. Neural network error squares diagram

Figure 3 shows the results of the real model and the model implemented with the neural network. As can be seen, the prediction estimated by the neural network is so close to the actual result that the actual and estimated output in most cases coincides. 


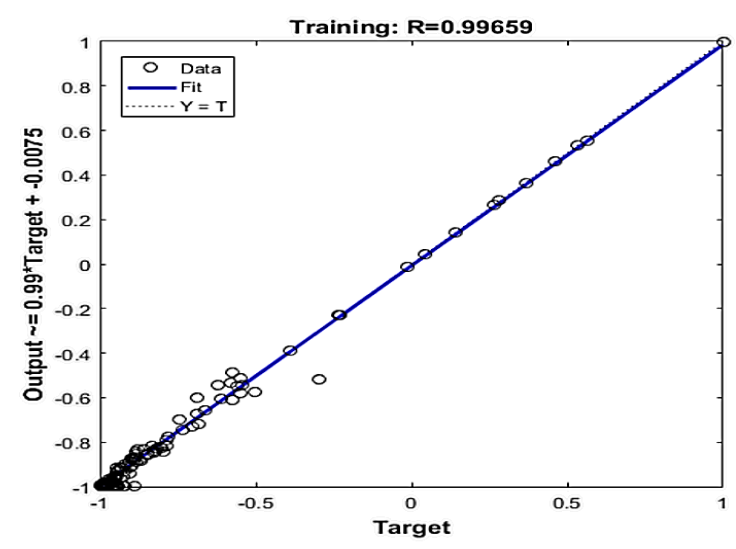

Figure 3. Regression equation between the real and estimated model in neural network

Neural network results optimized by the Gray wolf algorithm.

In this section, the weights of the neural network that has been implemented have been optimized by the Gray wolf algorithm as you can see in Table 4.

Table 4. Validation of assessment with different test and training data for neural network and neural network found with GWO

\begin{tabular}{cccccc}
\multirow{2}{*}{ Test data } & \multirow{2}{*}{ Train Data } & \multicolumn{2}{c}{ ANN } & \multicolumn{2}{c}{ ANN-GWO } \\
\cline { 3 - 6 } & & MSE Train & MSE Test & MSE Train & MSE Test \\
\hline $20 \%$ & $80 \%$ & $1.704^{-4}$ & 0.0066 & $1.405^{-6}$ & 0.0053 \\
\hline
\end{tabular}

The Gray Wolf Algorithm, which has the best mean square error in 122 generations with an initial population of 150 wolves. Also, the convergence of the Gray wolf algorithm in 122 generations can be seen in Figure 4.

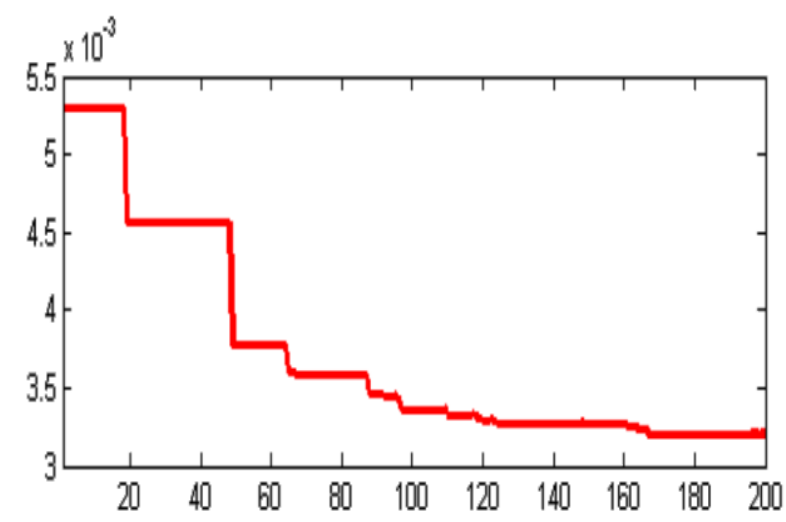

Figure 4. GWO Convergence Chart

Figures 5 and 6 show the performance of the neural network with the genetic algorithm in the training and testing phase. As can be seen in the test part of this algorithm has a good performance and the scattering rate is reduced. 


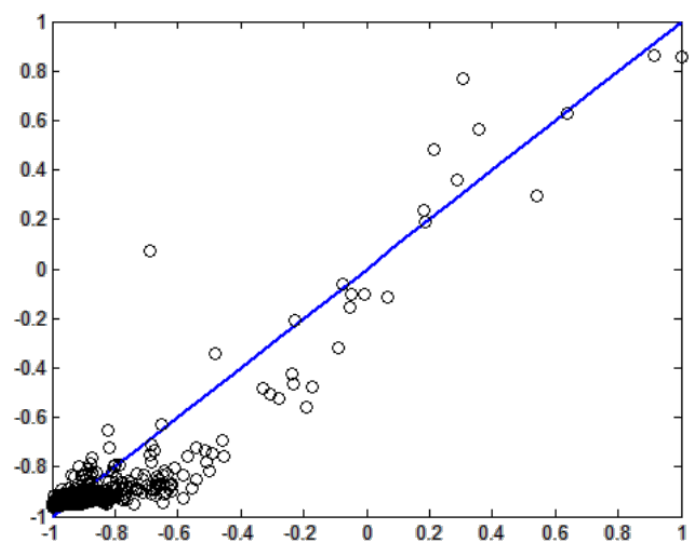

Figure 5. Comparison discharge of observational and computational flow in the training stage in neural network and Gray wolf algorithm

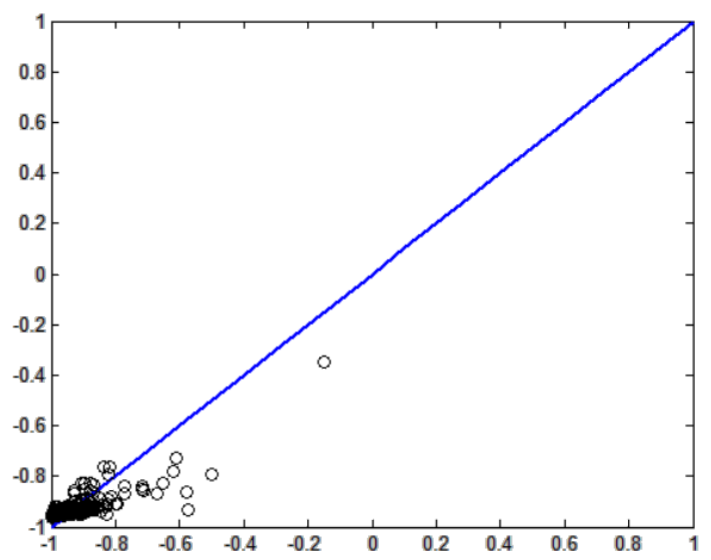

Figure 6. Comparison of observational and computational discharge predictions in the test phase in neural network and Gray wolf algorithm

This method is used to obtain the best amount of data for testing and training. As the results, the best rate is $80 \%$ for training and $20 \%$ for testing. Obtained results show the neural network error squares with $80 \%$ of the training data and $20 \%$ of the test data.

\section{Conclusions}

Flood has been one of the topics of interest to researchers, one of the methods of predicting it is to use the basic model of neural network. Also, various methods have been used to improve the neural network. In this study, the Gray wolf algorithm has been used and implemented. The results of the study showed that the Gray wolf algorithm has higher accuracy than other algorithms and it can also be said that a large amount of data of this algorithm compared to other algorithms is a significant point. Which makes it more accurate. The advantages of this research include the following:

- According to the results, the Gray wolf competition algorithm has a higher accuracy than the other algorithms studied. As a result, it can be used as a powerful tool in optimizing the weights of artificial neural networks.

- A validation technique can be used to make the answer reliable.

- Using the flow and river flow parameters, which are two important and reliable parameters for flood prediction. 
- Use a large database that increases implementation accuracy. In contrast to other studies, this number of data is very significant. The limitations of this algorithm are its time, which is high compared to some algorithms and good in others.

\section{References}

1. Abiodun, O. I., Jantan, A., Omolara, A. E., Dada, K. V., Mohamed, N. A., \& Arshad, H. (2018). State-of-the-art in artificial neural network applications: A survey. Heliyon, 4(11), e00938. (10.1016/j.heliyon. 2018. e00938)

2. Aoulmi, Yamina, et al. "Highly accurate prediction model for daily runoff in semi-arid basin exploiting Metaheuristic learning algorithms." IEEE Access 9 (2021): 92500-92515. (10.1109/ACCESS.2021.3092074)

3. Adib, A., \& Mahmoodi, A. (2017). Prediction of suspended sediment load using ANN GA conjunction model with Markov chain approach at flood conditions. KSCE Journal of Civil Engineering, 21(1), 447-457 . (10.1007/s12205-016-0444-2)

4. Adib, Arash, et al. "A new approach for suspended sediment load calculation based on generated flow discharge considering climate change." Water Supply (2021). (10.2166/ws.2021.069)

5. Akobeng, A. K. (2007). Understanding diagnostic tests 1 : sensitivity, specificity and predictive values. Acta paediatrica, 96(3), 338-3. (10.1111/j.1651-2227.2006.00180.x.)

6. Bakhtiari, Morteza; Almasi, Mohammad; Shahni Karamzadeh, Nima. (2010). Investigation of two artificial neural network learning algorithms in estimating suspended sediment load of river sediment. The first conference on applied research in Iranian water resources. Kermanshah: Kermanshah University of Technology. (In persian)

7. Berz, G. (2000). Flood disasters: lessons from the past-worries for the future. Paper presented at the Proceedings of the Institution of Civil Engineers-Water and Maritime Engineering.

8. Bozorg-Haddad, O., Zarezadeh-Mehrizi, M., Abdi-Dehkordi, M., Loáiciga, H. A., \& Mariño, M. A. (2016). A self-tuning ANN model for simulation and forecasting of surface flows. Water Resources Management, 30(9), 2907-2929. (10.1007/s11269-016-1301-2)

9. Bui, D. T., Pradhan, B., Nampak, H., Bui, Q.-T., Tran, Q.-A., \& Nguyen, Q.-P. (2016). Hybrid artificial intelligence approach based on neural fuzzy inference model and metaheuristic optimization for flood susceptibilitgy modeling in a high-frequency tropical cyclone area using GIS. Journal of Hydrology, 540, 317-330. (10.1016/j.jhydrol.2016.06.027)

10. Daliran, Firoozeh. (2014). Evaluation of flood effects using HEC-FIA software in Qahroud watershed. Master Thesis, Isfahan University of Technology. (In Persian)

11. Ehteram, Mohammad, et al. "Design of a hybrid ANN multi-objective whale algorithm for suspended sediment load prediction." Environmental Science and Pollution Research 28.2 (2021): 1596-1611. (10.1007/s11356-020-10421-y)

12. Fast, Laurent. (2013). Basics of Neural Networks: Structure, Algorithms, and Applications. Translated by Weiss, Hadi; Mafakheri, Kobra; Bagheri Shooraki, Saeed. Tehran: Nas Publications, 488 pages. (In Persian)

13. Heidari, E., Sobati, M.A. and Movahedirad, S., 2016. Accurate prediction of nanofluid viscosity using a multilayer perceptron artificial neural network (MLP-ANN). Chemometrics and intelligent laboratory systems, 155, pp.73-85. 10.1016/j.chemolab.2016.03.031

14. Heydari, Ali; Emami, Kamran; Mirni, Mohammad Hussein; Taqi Khan, Shahindokht; Moradi Fallah, Shadi; Barkhordar, Mehrdad. (2005). Flood forecasting and warning. Tehran, National Committee for Irrigation and Drainage of Iran. (In Persian)

15. Latt, Z. Z. (2015. (Application of feedforward artificial neural network in Muskingum flood routing: a black-box forecasting approach for a natural river system. Water Resources Management, 29(14), 4995-5014. (10.1007/s11269-015-1100-1)

16. Melesse, A., Ahmad, S., McClain, M., Wang, X., \& Lim, Y. (2011 .(Suspended sediment load prediction of river systems: An artificial neural network approach. Agricultural Water Management, 98(5), 855-866 (10.1016/j.agwat.2010.12.012)

17. Mirjalili, S., Mirjalili, S. M., Lewis, A. (2014). Grey Wolf Optimizer, Advances in engineering software, 69, 46-61.( 10.1016/j.advengsoft.2013.12.007) 
18. Ramezani, Farhad. (2011). Critique of meta-discovery methods in compositional optimization. Master Thesis. Faculty of Engineering, Nabi Akram University, Tabriz. (In Persian)

19. Seyed Abbasi, Mohammad, Goran Orimi, Mehdi; Farid Hosseini, Ali; Sharifi, Mohammad Baqir. (2011). Investigation of Cprecip parameter capability in order to determine the effect of snow on the prediction of daily river discharge by neural network and fuzzy neural network. Scientific Journal of Agriculture. Issue 35. (In persian)

20. Soomlek, C., Kaewchainam, N., Simano, T., \& So-In, C. (2015). Using backpropagation neural networks for flood forecasting in PhraNakhon Si Ayutthaya, Thailand. Paper presented at the 2015 International Computer Science and Engineering Conference (ICSEC). (10.1109/ICSEC. 2015.7401424)

21. Yadav, Arvind, Snehamoy Chatterjee, and Sk Md Equeenuddin. "Suspended sediment yield modeling in Mahanadi River, India by multi-objective optimization hybridizing artificial intelligence algorithms." International Journal of Sediment Research 36.1 (2021): 76-91. (10.1016/j.ijsrc.2020.03.018)

22. Zhang, Yu, and Yonghe Hao. "Loss prediction of mountain flood disaster in villages and towns based on rough set RBF neural network." Neural Computing and Applications (2021): 1-12. (10.1007/s00521-021-05902-1)

(C) 2021 by the authors. Licensee UAIC, Iasi, Romania. This article is an open access article distributed under the terms and conditions of the Creative Commons Attribution (CC BY-NC-ND) license (https://creativecommons.org/licenses/by-ncnd/4.0). 